\title{
COMPACT RIEMANN SURFACES WITH CONFORMAL INVOLUTIONS ${ }^{1}$
}

\author{
JANE GILMAN
}

\begin{abstract}
In this paper surfaces which have conformal involutions are characterized by their period matrices.
\end{abstract}

Let $S$ be a compact Riemann surface of genus $g, g \neq 0$, and $j$ a conformal involution on $S$ with $k$ fixed points, $k \neq 0$. Let $S^{\prime}$ be the factor surface and $g^{\prime}$ the genus of $S^{\prime}$. Then $g=2 g^{\prime}+k / 2-1$ by the RiemannHurwitz relation, so that $k$ is even.

PROPOSITION 1. S has a canonical homology basis consisting of homology classes of curves of the form

$$
\begin{aligned}
& a_{1}, \cdots, a_{g^{\prime}}, j\left(a_{1}\right), \cdots, j\left(a_{g^{\prime}}\right), c_{1}, \cdots, c_{k / 2-1}, \\
& b_{1}, \cdots, b_{g^{\prime}}, j\left(b_{1}\right), \cdots, j\left(b_{g^{\prime}}\right), d_{1}, \cdots, d_{k / 2-1},
\end{aligned}
$$

where $j\left(c_{i}\right) \sim-c_{i}$ and $j\left(d_{i}\right) \sim-d_{i}$ and where $\sim$ denotes homologous.

Proof. Let $P_{1}, \cdots, P_{k}$ be the fixed points of $j$ on $S$ and also their images on $S^{\prime}$. Let $(\boldsymbol{a}, \boldsymbol{b})$ be $2 g^{\prime}$ curves whose homology classes give a canonical homology basis for $S^{\prime}$. Let $n_{i}$ be a path on $S^{\prime}$ from $P_{i}$ to $P_{i+1}$ for all $i$ which does not intersect any curve in $(\boldsymbol{a}, \boldsymbol{b})$. Then it can be shown that $S$ is two copies of $S^{\prime}$ slit along the $n_{i}$ for $i$ odd and pasted together appropriately for some choice of slits $n_{i}$. If $c$ is any path on $S^{\prime}$, let $\tilde{c}$ be a lifting to $S$. Let $N_{i}=\tilde{n}_{i}-j\left(\tilde{n}_{i}\right)$ for $i=1,2, \cdots, k-1$. Let $d_{i}=N_{i}$ if $i$ is even; $d_{1}=N_{1} ;$ and define $d_{2 i+1}=d_{2 i-1}-N_{2 i+1}$ inductively. Since $j\left(N_{i}\right)=-N_{i}$, $j\left(\boldsymbol{d}_{i}\right)=-\boldsymbol{d}_{i}$ for all $i$. Let $c_{i}=\boldsymbol{d}_{\mathbf{2}}$ and $d_{i+1}=\boldsymbol{d}_{\mathbf{2}+1}$. Consider the set:

$$
\begin{gathered}
\tilde{\boldsymbol{a}}_{1}, \cdots, \tilde{\boldsymbol{a}}_{g^{\prime}}, j\left(\tilde{\boldsymbol{a}}_{1}\right), \cdots, j\left(\tilde{\boldsymbol{a}}_{g^{\prime}}\right), c_{1}, \cdots, c_{k / 2-1}, \\
\tilde{\boldsymbol{b}}_{1}, \cdots, \tilde{\boldsymbol{b}}_{g^{\prime}}, j\left(\tilde{\boldsymbol{b}}_{1}\right), \cdots, j\left(\tilde{\boldsymbol{b}}_{g^{\prime}}\right), d_{1}, \cdots, d_{k / 2-1} .
\end{gathered}
$$

Let $A \times B$ denote the intersection number of $A$ and $B$. Since $N_{i} \times N_{i+1}=1$ for all $i$, we have: $\tilde{\boldsymbol{a}}_{i} \times \tilde{\boldsymbol{b}}_{j}=\delta_{i j} ; \tilde{\boldsymbol{a}}_{i} \times j\left(\tilde{\boldsymbol{b}}_{j}\right)=0 ; \tilde{\boldsymbol{a}}_{i} \times c_{j}=0 ; \quad \tilde{\boldsymbol{a}}_{i} \times d_{j}=0$; $\tilde{\boldsymbol{a}}_{i} \times j\left(\tilde{\boldsymbol{a}}_{j}\right)=0 ; \tilde{\boldsymbol{b}}_{i} \times j\left(\tilde{\boldsymbol{b}}_{j}\right)=0$ and $c_{i} \times d_{j}=\delta_{i j}$ for all relevant $i$ and $j$. These

Received by the editors January 12, 1972.

AMS (MOS) subject classifications (1970). Primary 32G20.

1 This paper contains a part of the author's Ph.D. thesis written at Columbia University under the direction of Professor Lipman Bers whom the author wishes to thank for his help and encouragement.

(c) American Mathematical Society 1973 
intersection numbers can be used to show that these are $2 g$ homologously independent curves. Therefore, their homology classes must form a canonical homology basis for $S$.

Definition. A canonical homology basis of the form in Proposition 1 will be called a homology basis adapted to the involution $j$.

Proposition 2. Assume further that $k \neq 4$. Then $S$ has a conformal involution with $k$ fixed points if and only if it has a period matrix of the form $(I, Z)$ where

$$
Z=\left(\begin{array}{ccc}
A & B & C \\
B & A & -C \\
{ }^{t} C & -{ }^{t} C & K
\end{array}\right)
$$

where $A$ and $B$ are $g^{\prime} \times g^{\prime}$ symmetric, $C$ is $g^{\prime} \times(k / 2-1)$, and $K$ is $(k / 2-1) \times$ $(k / 2-1)$ symmetric. Further, $(I,(A+B))$ is a period matrix for $S^{\prime}$.

Proof. Pick a homology basis adapted to $j$. Let $w_{i}$ be the differential which has period 1 with respect to $a_{i}$ and period zero with respect to all other curves in line 1 . Let $j^{*}$ be the map which $j$ induces on differentials. Then $j^{*}\left(w_{i}\right)$ has period 1 with respect to $j\left(a_{i}\right)$ and period zero with respect to all other curves on line 1 . Let $w_{c_{i}}$ be the differential with period 1 with respect to $c_{i}$ and period zero with respect to all other curves in line 1 . Form the period matrix of $S$ with respect to these bases and use the fact that $\int_{j(x)} j^{*}(y)=\int_{x} y$ for any $x$ and $y$ to simplify the period matrix to the desired form.

Finally since $(\boldsymbol{a}, \boldsymbol{b})$ is a canonical homology basis for $S^{\prime}$ and since $\left(w_{i}+j^{*}\left(w_{i}\right)\right)$ as a $j$-invariant holomorphic differential defines a differential on $S^{\prime},(I,(A+B))$ is a period matrix for $S^{\prime}$.

To prove the converse we let $J_{g^{\prime}, k}$ be the matrix of the action of $j$ on a homology basis adapted to $j$ if $j$ is a conformal involution with $k$ fixed points. Then $Z$ is of the form shown in Proposition 2 if and only if $J_{g^{\prime}, k}(Z)=Z$, where $J_{g^{\prime}, k}$ now acts as an element of the (inhomogeneous) Siegel modular group. Indeed, if $J_{g^{\prime}, k}$, viewed as an element of $S_{p}(g, Z)$, acts on the homology basis, viewed as a column vector whose elements are arranged in the order of (1) then (2) of Proposition 1, then

where $M$ is $g \times g$ and

$$
J_{g^{\prime}, k}=\left(\begin{array}{l|l}
M & 0 \\
\hline 0 & M
\end{array}\right),
$$

$$
M=\left(\begin{array}{c|c|c}
0 & I_{g^{\prime}} & 0 \\
\hline I_{g^{\prime}} & 0 & 0 \\
\hline 0 & 0 & -I_{k / 2-1}
\end{array}\right),
$$


$I_{g^{\prime}}, I_{k / 2-1}$ being the indicated identity matrices; and then

$$
J_{g^{\prime}, k}(Z)=M Z M
$$

from which the form of $Z$ follows.

$J_{g^{\prime}, k}(Z)=Z$ implies (see [2, p. 28]) $S$ has a conformal involution whose action on homology is either given by $J_{g^{\prime}, k}$ or $-J_{g^{\prime}, k}$. If the latter occurs, apply the main result of [1] to conclude that either $g=0$, contrary to assumption, or $k$ is less than 4 .

In either case, $S$ has a conformal involution and we can apply either the Atiyah-Singer index theorem or the Lefschetz fixed point formula to show that the number of fixed points is $2-\operatorname{tr} J_{g^{\prime} . k}=k$ in the one case and $2-\operatorname{tr}\left(-J_{g^{\prime}, k}\right)=4-k$ in the other case. If $k$ is greater than 4 , the first case occurs; $k=4$ is excluded; and if $k=2$, whichever case occurs, the involution has 2 fixed points.

\section{REFERENCES}

1. Robert D. Accola, Automorphisms of Riemann surfaces, J. Analyse Math. 18 (1967), 1-5. MR 35 \#4398.

2. H. E. Rauch, A transcendental view of the space of algebraic Riemann surfaces, Bull. Amer. Math. Soc. 71 (1965), 1-39. MR 35 \#4403.

Department of Mathematics, State University of New York, Stony Brook, NEW YORK 11790

Current address: Department of Mathematics, Rutgers University, Newark Campus, Newark, New Jersey 07102 